NOTA TÉCNICA

\title{
ANÁLISIS QUÍMICO Y DIGESTIBILIDAD “IN VITRO” DE RASTROJO DE MAÍZ (Zea mays L.) ${ }^{1}$
}

\author{
Jesús Fuentes ${ }^{2}$, Calixtro Magaña ${ }^{4}$, Lorenzo Suárez ${ }^{2}$, Rodolfo Peña ${ }^{2}$, Sergio Rodríguez ${ }^{2}$, \\ Benjamín Ortíz de la Rosa ${ }^{3}$
}

\begin{abstract}
RESUMEN
Análisis químico y digestibilidad "in vitro" de rastrojo de maíz (Zea mays L.). Este experimento se llevó a cabo con el objetivo de evaluar el efecto del tratamiento físico y químico en la composición química y digestibilidad "in vitro" de la materia seca (DIVMS) y materia orgánica (DIVMO) de rastrojo de maíz sometido a dichos tratamientos. Setenta y cinco pacas de rastrojo de maíz (25 molidas, 25 picadas y 25 enteras) fueron tratadas con $4 \%$ de amoniaco anhidro $\left(\mathrm{NH}_{3}\right)$ con base en el peso seco durante cuatro semanas. El mismo número de pacas con igual tamaño de partícula permaneció sin tratar con $\mathrm{NH}_{3}$. Se utilizó un diseño experimental completamente al azar con arreglo factorial $2 \mathrm{x} 3$ (cero y cuatro $\% \mathrm{NH}_{3}$ y tres tamaños de partícula). Se observó un decremento en la cantidad de materia seca a medida que el tamaño de partícula aumentó. El incremento en el porcentaje de proteína cruda en el rastrojo tratado molido, picado y entero fue de 110, 116 y 91 unidades mayor respecto al rastrojo sin tratar. El extracto etéreo se vio incrementado por la amonificación en promedio 7,3\% con respecto al rastrojo sin tratar. El contenido de cenizas se incrementó en promedio 6,0\% con la amonificación. El $\mathrm{NH}_{3}$ disminuyó los valores de fibra detergente neutro en 20,0; 7,0 y 7,7\% para forraje molido, picado y entero, respectivamente. En lo que respecta a la fibra ácido detergente se observó un decremento de 7,17; 12,83 y $11,42 \%$ para el rastrojo molido, picado y entero, respectivamente, en relación al rastrojo sin tratar. La DIVMS y DIVMO se incrementaron con el tratamiento con $\mathrm{NH}_{3}$.
\end{abstract}

\begin{abstract}
Chemical analysis and in vitro digestibility of corn stover (Zea mays L). This experiment was conducted to evaluate the effects of chemical and physical treatments on the chemical composition and in vitro dry matter (IVDMD) and organic matter (IVOMD) digestibility of corn stover. Seventy five bales of corn stover (25 ground, 25 chopped, and 25 whole) were treated with $4 \%$ ammonia $\left(\mathrm{NH}_{3}\right)$ of the dry matter weight. A completely randomized design with factorial array $2 \times 3\left(0.0\right.$ and $4.0 \% \mathrm{NH}_{3}$ and three particle sizes). Results indicated that dry matter content decreased as particle size increased. Protein content increased by 110, 116 and 91 units for the ground, chopped and whole corn stover treated with $\mathrm{NH}_{3}$ with respect to the control. Ether extract increased by $7.3 \%$ for corn stover treated with $\mathrm{NH}_{3}$. Similar trend was followed by the ash content which increased by $6.0 \%$ for corn stover treated with $\mathrm{NH}_{3}$. Ammonia treatment affected negatively the neutral detergent fiber values by 20.0 , 7.0 and $7.7 \%$ for ground, chopped and whole corn stover, respectively. Similar results were found for acid detergent fiber with values of $7.17,12.53$ and $11.42 \%$ lower for ground, chopped and whole corn stover, respectively; than those found for the untreated material. IVDMD and IVOMD were increased with $\mathrm{NH}_{3}$ treatment.
\end{abstract}

\section{INTRODUCCIÓN}

En México es común la utilización del rastrojo de maíz como alimento para rumiantes, pese a que posee poco valor alimenticio, baja digestibilidad y es muy tosco debido a su estado de lignificación. El interés por la utilización de residuos agrícolas en la alimentación de rumiantes se ha venido incrementando en el ámbito

\footnotetext{
1 Recibido para publicación el 2 de abril del 2000. Presentado en la XLVI Reunión Anual del PCCMCA. San Juan, Puerto Rico. 2000.

2 UAAAN, Buenavista, Saltillo, Cohauila, México. E-mail: jesus_fuentes@hotmail.com.

3 ITA, Conkal, Yucatán, México.

4 Tesista UAAAN.
} 
mundial en los últimos años, a medida que la disponibilidad de granos se reduce. Es también importante la competencia nula entre monogástricos y rumiantes por alimentos fibrosos y, la habilidad que tienen los rumiantes para convertir esos materiales fibrosos en productos útiles para el hombre (carne, leche, pieles, lana, etc.). En México se producen alrededor de 70 millones de toneladas de residuos agrícolas de las cuales el rastrojo de maíz, de sorgo y paja de trigo, representan 58,$0 ; 12,0$ y $15,0 \%$, respectivamente (INEGI 1997). Existen métodos para tratar los forrajes fibrosos que permiten incrementar el consumo y la digestibilidad de estos alimentos y por lo tanto incrementar la productividad animal. Los mejores resultados se han obtenido con la aplicación de substancias alcalinas como amoníaco $\left(\mathrm{NH}_{3}\right)$, hidróxido de sodio $(\mathrm{NaOH})$ y urea $\left(\mathrm{NH}_{3}\right)$ $\mathrm{CO}_{3}$ (Asmud y Lars 1983, Klopfenstein 1980). En términos generales, el modo de acción de los álcalis es a través de la ruptura de las paredes celulares por medio de la solubilización de la hemicelulosa, la hidrólisis de los ésteres de ácidos urónicos y acéticos, aumentando la tasa de digestión ruminal de la celulosa al sufrir ésta un aumento en sus dimensiones y exponer mayor superficie al ataque de los microorganismos del rumen (Klopfenstein 1980). Al tratar el rastrojo con estas sustancias, se realiza una predigestión, por lo que se aprovecha de un modo más eficiente, ya que al hacerlo más disponible a los microorganismos del rumen se mejora la producción animal, con un costo menor de producción, ya que estos alimentos no son caros y las técnicas y materiales utilizados son sencillos. Otro aspecto importante en la producción animal es el determinar el tamaño óptimo del rastrojo para que pueda ser aprovechado por los microorganismos del rumen después de haber sido sometidos al tratamiento químico, por lo tanto los objetivos del presente trabajo fueron: evaluar el efecto del amoníaco $\left(\mathrm{NH}_{3}\right)$ anhídro sobre el rastrojo de maíz con diferente tamaño de partícula (molido, picado y entero) mediante análisis proximal y la determinación de la digestibilidad in vitro de la materia seca (DIVMS) y materia orgánica (DIVMO) de dicho rastrojo de maíz.

\section{MATERIALES Y MÉTODOS}

El presente estudio se llevó a cabo de mayo a agosto de 1998 en las instalaciones de la Universidad Autónoma Agraria Antonio Narro, localizada en Buenavista, Saltillo, Coahuila, México, ubicada a $25^{\circ} 22^{\prime}$ de latitud Norte y $101^{\circ} 01^{\prime}$ ' de longitud Oeste a $1742 \mathrm{msnm}$. Su clasificación climática es BWh xw(e), la cual tiene las siguientes características: muy seco, cálido, lluvias escasas todo el año y extremoso, con una precipitación media anual de $298,5 \mathrm{~mm}$. La temperatura media anual es de $14,80^{\circ} \mathrm{C}$, con una mínima de $11,90^{\circ} \mathrm{C}$ y una máxima de $21,30^{\circ} \mathrm{C}$ (Mendoza 1983). El experimento inició con el molido (criba de $2,5 \mathrm{~cm}$ ) y picado (cortes de $10 \mathrm{~cm}$ ) del rastrojo de maíz. Posteriormente 75 pacas ( 25 molidas, 25 picadas y 25 enteras) fueron tratadas con amoníaco anhídro mediante una modificación al método de Sundstol y Coxworth (1984), por un período de cuatro semanas. La cantidad fue de $4 \%$ peso en base seca. Igual número de pacas con los mismos tamaños de partícula $\mathrm{NH}_{3}$ inyectado permanecieron sin tratar. Se realizó el análisis proximal de acuerdo a la AOAC (1984), La fibra neutro detergente (FND) y fibra ácido detergente (FAD) se determinó de acuerdo a Van Soest (1970). La DIVMS y la DIVMO se determinaron por el método de Tilley y Terry modificado por Barnes (1970). Se utilizó un diseño experimental completamente al azar con arreglo factorial $2 \times 3$ (cero y cuatro $\%$ $\mathrm{NH}_{3}$ y tres tamaños de partícula) según Ostle (1965).

\section{RESULTADOS Y DISCUSIÓN}

El contenido de materia seca disminuyó a medida que se incrementó el tamaño de partícula para el rastrojo tratado con $\mathrm{NH}_{3}, 78,38 ; 76,30$ y $73,40 \%$ para el rastrojo molido, picado y entero, respectivamente. La materia seca del rastrojo sin tratar fue de 95,80\% (Cuadro 1). Se observó un incremento en el porcentaje de proteína cruda (PC) en el rastrojo de maíz tratado molido, picado y entero de 110,$0 ; 116,0$ y $91,0 \%$ al compararlo con el rastrojo sin tratamiento. Estos valores son menores a los obtenidos por Duarte y Shimada (1984), quienes encontraron un incremento de $148 \%$ de PC al tratar con $\mathrm{NH}_{3}$ rastrojo de maíz molido. Martínez et al. (1985) obtuvieron un $132 \%$ de incremento de PC al tratar con $\mathrm{NH}_{3}$ rastrojo de maíz en pacas. Resultados similares fueron indicados por Saenger et al. (1980) que encontraron un incremento de $157 \%$ al tratar rastrojo de maíz con $\mathrm{NH}_{3}$. Con relación al efecto del tratamiento químico sobre el contenido de extracto etéreo, la amonifica-

Cuadro 1. Contenido nutricional de rastrojo de maíz tratado con amoníaco anhidro $\left(\mathrm{NH}_{3}\right)$ con diferente tamaño de partícula. UAAAN, Saltillo, Coah., México. 1998.

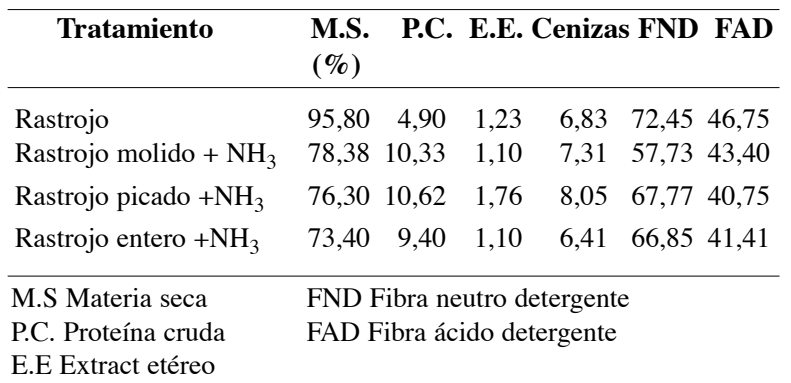


ción incrementó en 7,3\% esta fracción al compararlo con el rastrojo sin tratar, lo que coincide con resultados de García (1980), que encontró incrementos de 9,5\%, y Urrutia et al. (1982) que encontraron incrementos de $10,0 \%$. El contenido de cenizas se incrementó en promedio 6,0\%, al compararlo con el rastrojo sin tratar. Estos valores son inferiores a los mencionados por Herrera et al. (1982). Se encontró que el tratamiento con $\mathrm{NH}_{3}$ disminuyó en 20,0; 7,0 y 7,7\% el contenido de FND en el rastrojo molido, picado y entero, respectivamente al compararlo con el rastrojo sin tratamiento. Esto se debió aparentemente a que el $\mathrm{NH}_{3}$ solubilizó parte de la hemicelulosa, lo que hace más disponible el contenido celular. Estos valores son muy similares a los indicados por Shimada (1983) y Martínez et al. (1985), al tratar pacas de paja de trigo con $\mathrm{NH}_{3}$, los cuales tuvieron incrementos de 78,32 a $63,12 \%$. En lo que respecta a la FAD se observó un decremento de 7,17; 12,83 y $11,42 \%$ para el rastrojo molido, picado y entero, respectivamente, en relación al rastrojo sin tratar, lo cual difiere de los resultados obtenidos por Herrera et al. (1982), que encontraron incrementos de $10,6 \%$ en pacas de trigo tratados con $\mathrm{NH}_{3}$ en relación con las no tratadas. En el análisis de la DIVMS se encontraron incrementos a medida que se aumentaba el tamaño de partícula. Los valores encontrados fueron de 66,$05 ; 71,50$ y $71,94 \%$ para el rastrojo molido, picado y entero, respectivamente, y de $64,67 \%$ para el rastrojo sin tratar. Similares resultados fueron obtenidos por Llamas et al. (1985), Knapp et al. (1975) y Klopfenstein (1980). La DIVMO para el rastrojo sin tratar fue de $60,91 \%$, mientras que para el rastrojo tratado fue de 60,$38 ; 63,19$ y $64,10 \%$ para el rastrojo molido, picado y entero, respectivamente, lo cual coincide con los resultados de Paterson et al. (1981) y Nelson et al. (1984), pero contrasta con los obtenidos por de Morris y Mowat (1980), quienes encontraron una disminución de 5,0\%.

Cuadro 2. Digestibilidad in vitro de materia seca (DIVMS) y materia orgánica (DIVMO) de rastrojo de maíz tratado con amoníaco anhidro $\left(\mathrm{NH}_{3}\right)$. UAAAN, Saltillo, Coah., México. 1998.

\begin{tabular}{lcc}
\hline \multicolumn{1}{c}{ Tratamiento } & $\begin{array}{c}\text { DIVMS } \\
(\mathbf{\%})\end{array}$ & $\begin{array}{c}\text { DIVMO } \\
(\boldsymbol{\%})\end{array}$ \\
\hline Rastrojo sin tratamiento & 64,67 & 60,91 \\
Rastrojo molido + amoníaco & 66,05 & 60,38 \\
Rastrojo picado + amoníaco & 71,50 & 63,19 \\
Rastrojo entero + amoníaco & 71,94 & 64,10 \\
\hline
\end{tabular}

\section{CONCLUSIONES}

Los tratamientos físicos y químicos de forrajes toscos incrementan el valor nutritivo de los mismos, por lo que se debe de promover la utilización de estos tratamientos para poder tener una utilización óptima de este tipo de alimentos, que permitan reducir costos de alimentación y una mejor producción animal.

\section{LITERATURA CITADA}

AOAC (Association of Official Agricultural Chemist). 1984. Official methods of analysis. 14th Edition. AOAC. Washington, D.C. USA.

ASMUD, E.; LARS, V.M. 1983. Forrajes conservados como alimento para vacas lecheras. In: Broster, W.; Henry, S. eds. Estrategia de alimentación para vacas lecheras de alta producción. México. p. 258.

BARNES, R.F. 1970. Collaborative research with the two stage in vitro rumen fermentation technique. In Barnes, R.; Clanton, D; Gordon, C.; Klopfenstein, T.; Waldo, D. eds. Proceedings of the National Conference of Forage Quality Evaluation and Utilization. Lincoln, Nebraska, USA. p. 235-254.

DUARTE, J.A.; SHIMADA, A. 1984. Comportamiento del borrego pelibuey en crecimiento alimentado con dietas con base en rastrojo de maíz tratado con álcalis $\left(\mathrm{NH}_{3}\right.$, $\mathrm{NaOH}$ y urea). Tec. Pec. Mex. 47:141-146.

GARCÍA, E.R. 1980. Alimentación de borregos criollos con raciones a base de rastrojo de maíz con diferentes niveles de hidróxido de sodio $(\mathrm{NaOH})$. Tesis Maestría. Universidad Autónoma Agraria Antonio Narro (UAAAN). Saltillo, Coahuila, México. p. 76.

HERRERA, S.R.; CHURCH, D.C.; KELLEMS, R.O. 1982. The effect of ammoniation treatment on intake on nutritive value of wheat straw. J. Anim. Sci. 54:603-608.

INEGI ( Instituto Nacional de Estadística, Geografía e Informática). 1997. El sector agroalimentario en México. Edición 1997. México, INEGI. p. 340.

KLOPFENSTEIN, T. 1980. Increasing the nutritive value of crop residues by chemical treatment. Animal Science Dept. University of Nebraska. Lincoln, Nebraska, USA. 81 p.

KNAPP, W.R.; HOLT, D.A.; LECHTENBERG, V.L. 1975. High preservation and quality improvement by anhydrous ammonia treatment. Agronomy J. 67:766-769.

LLAMAS, L.G.; CAÑEZ, H.; GÓMEZ,R.; DÍAZ, T. 1985. Uso de paja de trigo tratada con amoníaco en la alimentación de novillos en crecimiento en corral de engorda. Tec. Pec. Mex. 46:46-53.

MARTÍNEZ, A.A.; SORIANO, M.J.; SHIMADA, A. 1985. Crecimiento de borregos pelibuey alimentados con rastrojo de maíz tratado con amoníaco anhídro. Tec: Pec. Mex. 48:54-65. 
MENDOZA, H.J. 1983. Diagnóstico climático para la zona de influencia de la UAAAN. Universidad Autónoma Agraria Antonio Narro. Saltillo, Coahuila, México. p. 615.

MORRIS, P.J; MOWAT, D.N. 1980. Nutritive value of ground and or ammoniated corn stover. Can. J. Anim. Sci. 60:327-336.

NELSON, M.L.; KLOPFENSTEIN, T.; BRITTON, R.A. 1984. Protein supplementation of ammoniated roughages. 1. Corncobs supplemented with a blood meal-corn gluten meal mixture lamb studies. J. Anim. Sci. 59:160169.

OSTLE, B. 1965. Statistics in research. Ames, Iowa, USA. Iowa State University Press. 280p.

PATERSON, J.A.; KLOPFENSTEIN, T.; BRITON, R.A. 1981. Ammonia treatment of corn plant residues: digestibility and growth rates. J. Anim. Sci. 53:1952-1600.
SAENGER, P. R.; LEMANAGER; HENDRIX, K. 1980. Intake and digestibility of corn harvest residue treated with anhydrous ammonia. J. Anim. Sci. 59:1601-1609.

SHIMADA, A. 1983. Fundamentos de nutrición animal comparativa. Consultores en producción animal. México. p. 272 .

SUNDSTOL, F.; COXWORTH, E. 1984. Ammonia treatment. In: Suntol, F.; Owen, E. eds. Straw and other fibrous by products as feed. Elsevier. Netherlands. p. 197-247.

URRUTIA, M.; MARTÍNEZ, L.; SHIMADA, A. 1982. Valor nutritivo de rastrojo y ensilaje de maíz con o sin mazorca tratados con hidróxido de sodio para borregos en crecimiento. Tec. Pec. Mex. 42:7-16.

VAN SOEST, P. J. 1970. The chemical basis for nutritive evaluation of forages. Proc. Nat. Conf. on Forage Quality Evaluation and Utilization. Lincoln, Nebraska, USA. 78p. 